\title{
DIFERENCIAÇÃo ENTRE Egeria densa E Egeria najas PELOS Caracteres ANatômicos Foliares ${ }^{1}$
}

\author{
Differentiation Between Egeria densa and Egeria najas by Leaf Anatomic Characters
}

RODELlA, R.A. ${ }^{2}$, COSTA, N.V. ${ }^{3}$, COSTA, L.D.N.C. ${ }^{4}$ e MARTINS, D. ${ }^{5}$

\begin{abstract}
RESUMO - Dentre as plantas daninhas aquáticas imersas de maior importância nos reservatórios de usinas hidrelétricas e em represas de pequeno porte no Brasil, destacam-se as espécies Egeria densa e Egeria najas, cuja identificação pode ser difícil na fase vegetativa. O objetivo deste trabalho foi diferenciar cinco acessos de E. densa e três de E. najas, coletados nos reservatórios de Jupiá, Salto Grande, Três Irmãos, Promissão, Nova Avanhandava e Ibitinga, do complexo da Companhia Energética de São Paulo (CESP) do Estado de São Paulo, quanto às características anatômicas descritivas e quantitativas do limbo foliar, procurando-se obter melhor entendimento sobre as relações dessas estruturas anatômicas com a penetração e translocação de herbicidas, além de auxiliar na identificação de acessos suscetíveis e resistentes a determinado produto químico. Amostras do terço médio do limbo foram fixadas em FAA 50, cortadas transversalmente em micrótomo rotatório com $8 \mu \mathrm{m}$ de espessura e coradas com azul-de-toluidina. Foi analisada a estrutura foliar e foram quantificados os caracteres anatômicos da nervura central (\% epiderme das faces adaxial e abaxial, \% feixe vascular e \% parênquima) e da região situada entre a nervura e o bordo do limbo (\% epiderme das faces adaxial e abaxial e espessura da folha). Os dados das variáveis quantitativas foram submetidos aos testes estatísticos multivariados de Análise de Agrupamento e Análise de Componentes Principais. Houve formação de três grupos principais: o primeiro foi constituído pelos três acessos de E. najas; o segundo, por quatro acessos de E. densa; e o terceiro, por apenas um acesso de E. densa. O caráter que mais contribuiu para a diferenciação entre os acessos foi a $\%$ feixe vascular da nervura central, seguido da \% epiderme da face abaxial da nervura central e \% epiderme das faces adaxial e abaxial da região entre a nervura e o bordo foliar. Concluiuse que a utilização de caracteres anatômicos quantitativos permitiu auxiliar na diferenciação dos acessos e das espécies estudadas; entretanto, devem ser incrementados os estudos relacionando a estrutura anatômica com a resistência e suscetibilidade aos herbicidas.
\end{abstract}

Palavras-chave: Hydrocharitaceae, planta aquática, planta daninha, anatomia foliar.

\begin{abstract}
Seven leaf blade quantitative anatomic characters were used to differentiate five accesses of Egeria densa, and three accesses of Egeria najas, collected in 'Jupiá', 'Salto Grande', 'Três Irmãos', 'Promissão', 'Nova Avanhandava' and 'Ibitinga' reservoirs, in relation to leaf blade descriptive and quantitative anatomic characteristics, in order to obtain a better understanding the relations among these anatomic structures and herbicide penetration and translocation. Another objective was to provide identification of susceptible and resistant accesses to some chemical products. Leaf samples were collected, fixed in FAA 50, infiltrated in glycol metacrylate resin, cut transversally with $8 \mu \mathrm{m}$ of thickness, and stained with toluidine blue. The following leaf anatomic middle vein characters were quantitatively evaluated (\%): adaxial and abaxial epidermis, vascular bundle and parenchyma. In the region between the vein and the leaf margin, the \% adaxial and abaxial epidermis and leaf thickness were evaluated. The quantitative anatomic characters of the leaf were submitted to statistic multivariate methods of
\end{abstract}

Recebido para publicação em 19.8.2005 e na forma revisada em 5.5.2006.

2 Professor Assistente Doutor, Departamento de Botânica, Instituto de Biociências de Botucatu, UNESP, Caixa Postal 510, 18618-000 Botucatu-SP; ${ }^{3}$ Eng.-Agr., Doutorando em Agricultura, Faculdade de Ciências Agronômicas de Botucatu, UNESP; ${ }^{4}$ Graduanda em Agronomia, FCA-UNESP; ${ }^{5}$ Professor Adjunto, Departamento de Produção Vegetal, FCA-UNESP, Caixa Postal 237, 18603-970 Botucatu-SP.

Planta Daninha, Viçosa-MG, v. 24, n. 2, p. 211-220, 2006 
Cluster Analysis and Principal Components Analysis. Results showed that three groups were formed according to similarity levels: group 1 - three accesses of $\mathbf{E}$. najas; group 2 - four accesses of $\boldsymbol{E}$. densa; group 3 - one access of $\mathbf{E}$. densa. The anatomic characters with high discriminatory degree were: \% vascular bundle and abaxial epidermis of middle vein, and \% adaxial and abaxial epidermis of the region between the vein and leaf margin. In conclusion, it was observed that the quantitative anatomic characters were determinant in differentiating accesses and aquatic weed species; however, further studies must be conducted to relate the anatomical structures to resistance and susceptibility to herbicides.

Keywords: Hydrocharitaceae, aquatic plant, weed, leaf anatomy.

\section{INTRODUÇÃO}

As plantas aquáticas possuem importantes funções para a manutenção do equilíbrio dos ambientes aquáticos, pois atuam na proteção e estabilização das margens de rios e reduzem os efeitos da erosão. São fundamentais também na cadeia alimentar, na oxigenação da água e na absorção de nutrientes. Contudo, quando esse equilíbrio é alterado, pode ocorrer rápido e excessivo desenvolvimento dessas plantas aquáticas (Tanaka, 2000). Outras possíveis causas do desenvolvimento desordenado das espécies de plantas aquáticas são o lançamento de efluentes de origem urbana e/ou industrial e o transporte de fertilizantes utilizados na agricultura para os corpos hídricos, o que contribui para a eutrofização dos ambientes aquáticos.

A eutrofização dos sistemas aquáticos pode acarretar prejuízos à atividade pesqueira, à saúde pública, aos esportes náuticos, ao turismo e promover o entupimento de grades de tomadas de água das turbinas de usinas hidrelétricas, devido à alta produção de biomassa das populações de plantas aquáticas (Yeo et al., 1984; Tanaka, 1998). Do ponto de vista ambiental, algumas espécies de crescimento rápido podem suprimir outras menos agressivas, desejáveis para manutenção da diversidade, ou modificar negativamente algumas características físicas da água (Cardoso et al., 2002; Marcondes et al., 2003b).

Dentre as várias espécies que proliferam nos reservatórios das usinas hidrelétricas do Estado de São Paulo, destacam-se duas espécies imersas: Egeria densa e E. najas, da família Hydrocharitaceae. Ambas são originárias da América do Sul e multiplicam-se, principalmente, por fragmentação do caule, sendo a reprodução por sementes muito rara (Cook \& Urmi-König, 1984; Bowmer et al., 1995; Kissmann \& Groth, 1997).

A comercialização da egéria, como planta ornamental para aquários, viabilizou sua disseminação por várias partes do mundo, sendo encontrada no Japão e nos Estados Unidos, onde se distribui pela Califórnia, Arizona, Washington e Flórida (Bowmer et al., 1995; Roberts et al., 1999; Martins et al., 2003).

O reservatório da Usina Hidrelétrica Engo Souza Dias (Jupiá), da Companhia Energética de São Paulo (CESP), apresenta grandes populações de plantas aquáticas imersas, principalmente de E. densa, E. najas e Ceratophyllum demersum. Essas espécies ocupam grandes extensões do reservatório, com acúmulos de matéria fresca de até $50 \mathrm{t} \mathrm{ha}^{-1}$ nos locais de maior colonização. No período chuvoso, as cheias deslocam massas de plantas em direção à usina, onde se acumulam nas grades de proteção das unidades geradoras, tornando obrigatória sua remoção, a fim de evitar o entupimento das grades (Marcondes et al., 2003b). O prejuízo não se resume aos custos com a coleta e transporte das plantas e com a substituição de grades, mas também à perda potencial de receitas devido à redução na geração de energia. Em reservatório de pouca capacidade de armazenamento de água, essa redução de potência leva a uma sobra de água que deve ser vertida, sem gerar energia elétrica (Marcondes et al., 2003a). Thomaz (2002) afirma que a construção de reservatórios e a manipulação dos niveis higrométricos podem estimular o desenvolvimento indesejado de plantas aquáticas. 
A morfologia das plantas, principalmente os caracteres anatômicos, pode determinar o grau de facilidade ou de dificuldade de absorção dos herbicidas pelas folhas. Dessa forma, o estudo da anatomia do limbo foliar de plantas daninhas destaca-se como uma técnica importante na descrição de estruturas que podem influenciar a absorção dos herbicidas, além de auxiliar na identificação de espécies (Procópio et al., 2003a,b).

Ferreira et al. (2002) relatam que o estudo anatômico foliar pode melhorar o entendimento sobre as barreiras que cada espécie impõe à penetração dos herbicidas e, assim, fornecer subsídios para a busca de estratégias que superem tais obstáculos. Dessa maneira, a análise anatômica descritiva e quantitativa da folha pode ser aplicada com sucesso na identificação de espécies suscetíveis, tolerantes ou resistentes a determinado produto químico, contribuindo com os estudos de seletividade de herbicidas às espécies terrestres ou aquáticas.

As plantas de E. densa e E. najas são morfologicamente muito semelhantes, o que dificulta a identificação das espécies em levantamentos fitossociológicos realizados nos reservatórios das usinas hidrelétricas. A alta similaridade morfológica existente entre várias espécies pertencentes à família Hydrocharitaceae, como E. densa, E. najas, Elodea canadensis, Elodea nuttallii, Hydrilla verticillata e Lagarosiphon major, é ressaltada por vários autores (Solymosy \& Gangstad, 1974; Cook \& Urmi-König, 1984; Yeo et al., 1984; Bowmer et al., 1995; Hoyer et al., 1996).

Martins et al. (2003) citam que os estudos realizados com egéria sugerem a caracterização genética de acessos coletados no Brasil, para a possivel verificação da origem das plantas introduzidas nos Estados Unidos, Japão, Nova Zelândia e Europa, bem como para o auxílio no manejo químico, com enfoque em seleção e resistência a herbicidas.

Segundo Weiss et al. (1997), acessos coletados na Nova Zelândia de E. densa são geneticamente isolados e, possivelmente, foram introduzidos de uma população nativa da América do Sul. A caracterização de populações de plantas é de extrema importância para estabelecer planos de manejo apropriados, minimizar custos e garantir a repetição de resultados no campo (Mori et al., 1999).

Assim, o objetivo deste trabalho foi diferenciar oito acessos de espécies daninhas aquáticas imersas (E. densa e E. najas), coletadas nos reservatórios do complexo CESP do Estado de São Paulo, quanto às características anatômicas descritivas e quantitativas do limbo foliar, procurando-se obter melhor entendimento sobre as relações dessas estruturas anatômicas com a penetração e translocação de herbicidas, além de auxiliar na identificação de acessos suscetíveis e resistentes a determinado produto químico.

\section{MATERIAL E MÉTODOS}

Nas hidrelétricas do complexo CESP do Estado de São Paulo, plantas de Egeria densa foram coletadas nos reservatórios de Jupiá, Nova Avanhandava, Promissão e Salto Grande; e as de E. najas nos reservatórios de Ibitinga, Jupiá e Três Irmãos. A constituição genética dos acessos foi identificada pela técnica de RAPD, em estudo preliminar realizado por Martins et al. (2003), no Laboratório de Biologia Molecular do Núcleo de Pesquisas Avançadas em Matologia (NUPAM), da Faculdade de Ciências Agronômicas de Botucatu - UNESP. Foi encontrada no reservatório de Jupiá diferença genética entre dois acessos de $E$. densa, os quais foram identificados como Jupiá ${ }^{1}$ e Jupiá ${ }^{2}$.

A localização dos acessos (Tabela 1) foi determinada utilizando-se o aparelho de Sistema de Posição Geográfica (GPS), marca Garmin. A Figura 1 representa o mapa do Estado de São Paulo com a localização dos reservatórios, nos quais foram coletados os acessos de ambas as espécies de Egeria. Estes foram acondicionados, sob condições de campo, em caixas-d'água de fibra de vidro de 350 litros, contendo uma camada de $15 \mathrm{~cm}$ de solo no fundo.

O delineamento experimental utilizado foi o inteiramente casualizado, com oito tratamentos (três acessos de E. najas e cinco acessos de E. densa) e quatro repetições. O material foliar foi amostrado a $5 \mathrm{~cm}$ abaixo do ápice, quando as folhas se apresentavam totalmente expandidas, antes do florescimento. 
Tabela 1 - Coordenadas geográficas dos locais de coleta dos oito acessos de Egeria densa e E. najas presentes nos reservatórios das usinas hidrelétricas do complexo CESP, do Estado de São Paulo

\begin{tabular}{|c|c|c|c|c|c|c|}
\hline Acesso & Espécie & UTM & $22 \mathrm{~K}$ & Latitude & Longitude & Local \\
\hline Ibitinga & E. najas & 709478 & 7580732 & $\mathrm{~S} 21^{\circ} 51^{\prime} 54,1^{\prime \prime}$ & $\mathrm{W} 48^{\circ} 58^{\prime} 22,1^{\prime \prime}$ & Ribeirão Claro, MD \\
\hline Jupiá & E. najas & 456114 & 7713501 & $\mathrm{~S} 20^{\circ} 40^{\prime} 38,5^{\prime \prime}$ & W51 $21^{\circ} 16,9 "$ & Reservatório, Tietê, MD \\
\hline Três Irmãos & E. najas & 556673 & 7671509 & $\mathrm{~S} 21^{\circ} 03^{\prime} 23,2^{\prime \prime}$ & W50 27 ' $16,2 "$ & Montante da Ponte da SO-463, ME \\
\hline Jupiá $^{1}$ & E. densa & 456114 & 7713501 & $\mathrm{~S} 20^{\circ} 40^{\prime} 38,5^{\prime \prime}$ & W51 $251^{\circ} 16,9^{\prime \prime}$ & Reservatório, Tietê, MD \\
\hline Jupiá $^{2}$ & E. densa & 454743 & 7714202 & $\mathrm{~S} 20^{\circ} 40^{\prime} 15,6^{\prime \prime}$ & W51 $21^{\circ} 04,2 "$ & Reservatório, Tietê, MD \\
\hline Nova Avanhandava & E. densa & 623139 & 7644647 & $\mathrm{~S} 21^{\circ} 17^{\prime} 45,0^{\prime \prime}$ & W49 48'46,3” & Lagoa, ME \\
\hline Promissão & E. densa & 689490 & 7610733 & $\mathrm{~S} 21^{\circ} 35^{\prime} 47,0^{\prime \prime}$ & W49 10 ' $10,5^{\prime \prime}$ & Lagoa do Limoeiro, ME \\
\hline Salto Grande & E. densa & 603426 & 7467774 & $\mathrm{~S} 22^{\circ} 53^{\prime} 41,2^{\prime \prime}$ & W49 $59^{\prime} 29,8^{\prime \prime}$ & Meio do reservatório \\
\hline
\end{tabular}

UTM: Projeção Universal Transversal de Mercator (sistema métrico e cartesiano de coordenadas geográficas). 22K: Zona Meridiana.

MD: margem direita. ME: margem esquerda.

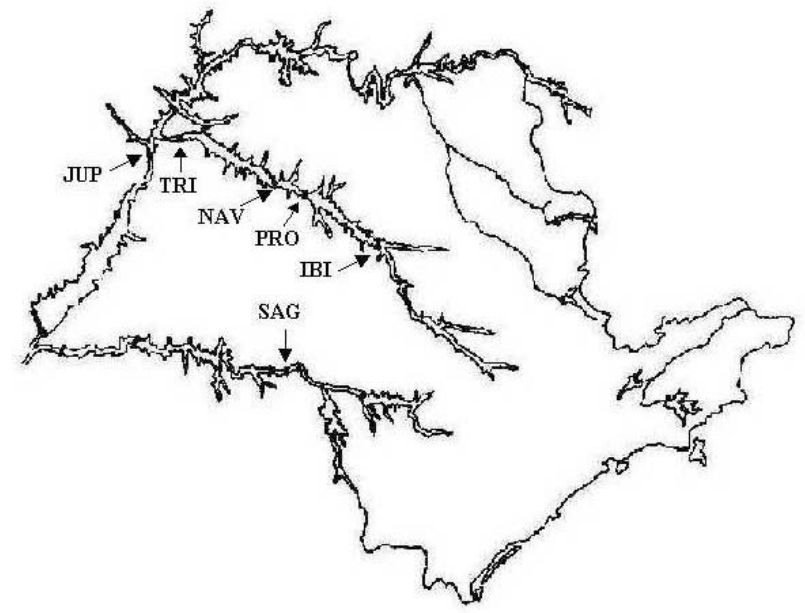

Figura 1 - Reservatórios do complexo CESP do Estado de São Paulo, nos quais foram coletados oito acessos de Egeria densa e E. najas. JUP: Jupiá, TRI: Três Irmãos, NAV: Nova Avanhandava, PRO: Promissão, IBI: Ibitinga e SAG: Salto Grande.

Para análise anatômica, foram tomadas porções da região mediana do limbo foliar, fixando-as em FAA 50 (formaldeído+ácido acético glacial+álcool 50\%) durante 48 horas, e, posteriormente, conservadas em álcool 70\% (Johansen, 1940).

Amostras da nervura central e da região situada entre a nervura e o bordo do limbo foliar foram infiltradas com historresina, conforme a técnica de Gerrits (1991). Em seguida, os materiais foram seccionados transversalmente em micrótomo rotatório, com espessura de $8 \mu \mathrm{m}$, e corados com azul-de-toluidina (O’Brien et al., 1964). A estrutura anatômica do limbo foi observada em microscopia de luz. Os limites e contornos dos tecidos foram desenhados com o auxílio de microscópio de projeção, sendo as áreas mensuradas por meio de mesa digitalizadora, usando-se um programa computacional específico (Souza et al., 2005). Os dados de áreas foram transformados em valores porcentuais em relação à área total de ambas as regiões amostradas.

Foram quantificados caracteres anatômicos do limbo foliar, na nervura central $(\%$ epiderme das faces adaxial e abaxial, \% feixe vascular e \% parênquima) e na região entre a nervura e o bordo do limbo (\% epiderme das faces adaxial e abaxial e espessura da folha).

Os valores dos sete caracteres anatômicos foram submetidos aos testes estatísticos multivariados de Análise de Agrupamento e Análise de Componentes Principais (Sneath \& Sokal, 1973), com a finalidade de discriminar os acessos e as espécies de Egeria de acordo com seu grau de similaridade. Na realização da Análise de Agrupamento empregou-se a Distância Euclidiana Média, com o algoritmo UPGMA ('Unweighted Pair Group Method with Arithmetic Average'), conforme recomendado por Rohlf (1970).

\section{RESULTADOS E DISCUSSÃO}

\section{Características anatômicas descritivas}

O limbo foliar de E. densa e E. najas apresenta apenas uma nervura distinta: a nervura central. Na Figura 2 são apresentadas as 
secções transversais do limbo, compreendendo o terço médio da nervura central (Figura 2A, B) e da região entre a nervura e o bordo foliar (Figura 2C, D) das duas espécies de plantas daninhas aquáticas estudadas.

A estrutura da nervura central de $E$. densa (Figura 2A) e E. najas (Figura 2B) apresenta: epiderme unisseriada em ambas as faces, recoberta por cutícula e apresentando cloroplastos; ausência de tricoma aculeado nas células epidérmicas da face abaxial, como também relataram Hoyer et al. (1996) para E. densa; escasso parênquima com células irregulares; e feixe vascular único com difícil distinção entre xilema e floema, como descreveram Yeo et al. (1984) para Hydrilla verticillata.

A região entre a nervura e o bordo do limbo foliar de E. densa (Figura 2C) e E. najas (Figura 2D) apresentam: epiderme unisseriada em ambas as faces e com cutícula mais espessada na face adaxial, como também constatou Sculthorpe (1967) em folhas de diversas espécies aquáticas, como Elodea canadensis; ocorrência de células epidérmicas com maiores dimensões na face adaxial do que na face abaxial; células epidérmicas contendo cloroplastos, desprovidas de estômatos e com espaços intercelulares entre as células das faces adaxial e abaxial; ausência de parênquima e de feixes vasculares, sendo o limbo constituído apenas por duas camadas de células, ou seja, a camada epidérmica das faces adaxial e abaxial; e ausência de fibras marginais e presença de idioblastos taniníferos no bordo do limbo em $E$. densa, características estas também observadas por Cook \& UrmiKönig (1984).

\section{Características anatômicas quantitativas}

Na Tabela 2 são apresentados os valores médios dos caracteres anatômicos quantitativos do limbo foliar, para os oito acessos de $E$. densa e E. najas, submetidos à análise estatística multivariada.

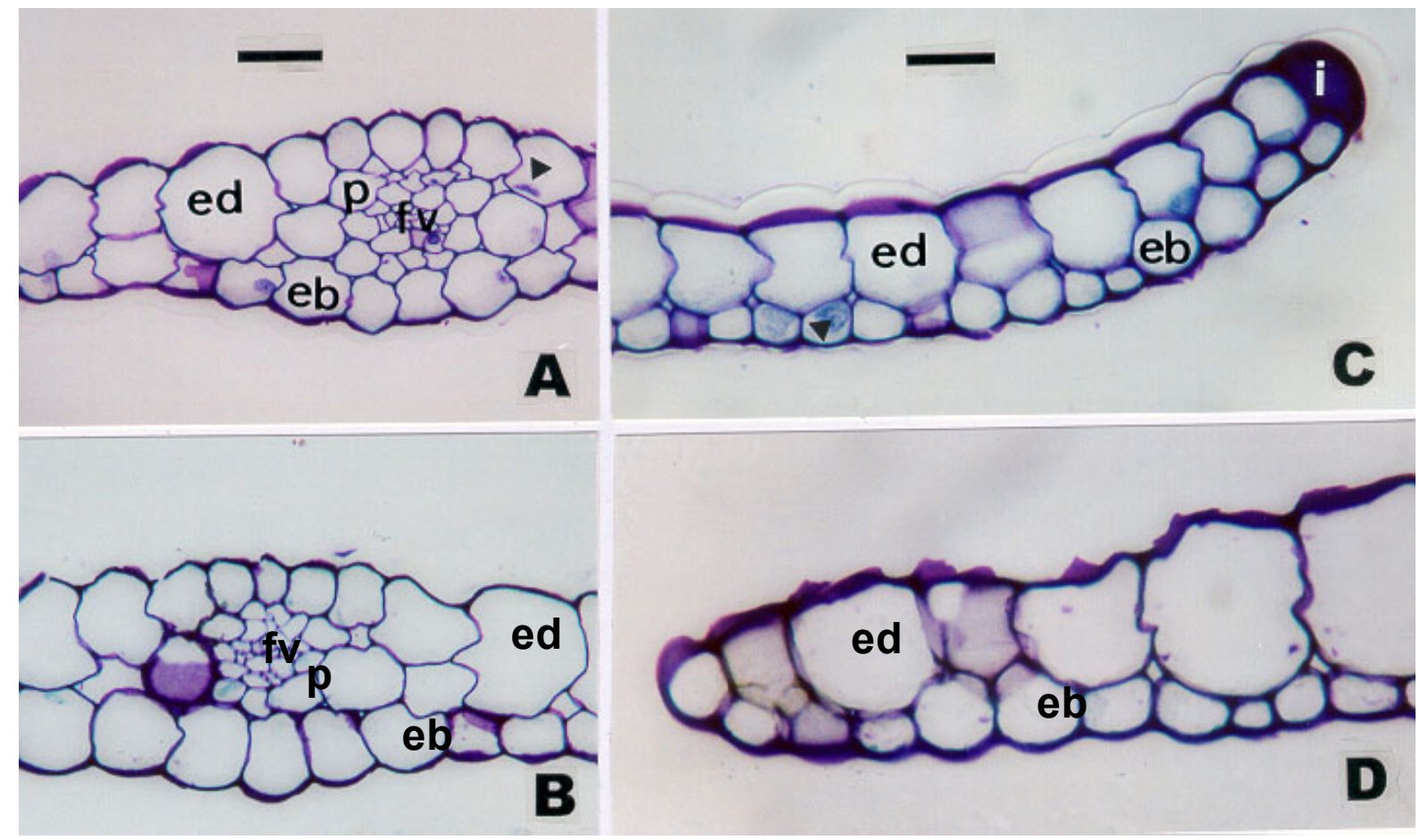

Figura 2 - Secções transversais do limbo foliar das espécies de Egeria. A. região da nervura central de E. densa; B: região da nervura central de E. najas; C: região entre a nervura e o bordo do limbo de E. densa; D: região entre a nervura e o bordo do limbo de E. najas; eb: epiderme da face abaxial; ed: epiderme da face adaxial; fv: feixe vascular; i: idioblasto taninífero; p: parênquima; e $\mathbf{\nabla}$ : cloroplasto. Barra $=46 \mu \mathrm{m}(\mathrm{A}-\mathrm{B})$ e $23 \mu \mathrm{m}(\mathrm{C}-\mathrm{D})$. 
O resultado da Análise de Agrupamento, representado na forma de dendrograma (Figura 3), permitiu agrupar os oito acessos das duas espécies de Egeria, de modo que aqueles pertencentes a um mesmo grupo apresentaram maior similaridade que os acessos de grupos diferentes. Os oito acessos constituíram três grupos principais, em nivel de 0,16 na escala de distância de similaridade, os quais apresentaram a seguinte disposição: grupo 1 - formado pelos três acessos de E. najas coletados nos reservatórios de Jupiá, Três Irmãos e Ibitinga; grupo 2 - constituído por quatro acessos de E. densa, coletados nos reservatórios de Jupiá ${ }^{1}$, Jupiá ${ }^{2}$, Salto Grande e Promissão; e grupo 3 - formado apenas por um acesso de E. densa, coletado no reservatório de Nova Avanhandava.

Tabela 2 - Valores médios dos caracteres anatômicos quantitativos do limbo foliar de oito acessos de Egeria densa e E. najas, coletados em reservatórios do complexo CESP do Estado de São Paulo

\begin{tabular}{|c|c|c|c|c|c|c|c|c|}
\hline \multirow{2}{*}{ Caráter } & \multicolumn{3}{|c|}{ E. najas } & \multicolumn{5}{|c|}{ E. densa } \\
\hline & IBI & JUP & TRI & $\mathrm{JUP}^{1 /}$ & $\mathrm{JUP}^{2 / !}$ & NAV & PRO & SAG \\
\hline & \multicolumn{8}{|c|}{ Região da Nervura Central } \\
\hline$\%$ Epiderme Face Adaxial & 41,73 & 42,66 & 41,51 & 53,29 & 47,88 & 41,70 & 41,16 & 41,20 \\
\hline \% Epiderme Face Abaxial & 30,79 & 35,10 & 38,59 & 28,06 & 29,24 & 29,74 & 27,85 & 32,98 \\
\hline \% Parênquima & 21,74 & 18,32 & 16,31 & 12,97 & 16,45 & 21,81 & 22,56 & 17,79 \\
\hline \multirow[t]{2}{*}{$\%$ Feixe Vascular } & 5,74 & 3,91 & 3,59 & 5,68 & 6,42 & 6,75 & 8,43 & 8,03 \\
\hline & \multicolumn{8}{|c|}{ Região entre a Nervura Central e o Bordo do Limbo } \\
\hline \% Epiderme Face Adaxial & 61,77 & 60,81 & 63,11 & 57,92 & 59,80 & 47,05 & 58,12 & 53,15 \\
\hline$\%$ Epiderme Face Abaxial & 38,23 & 39,19 & 36,89 & 42,18 & 40,20 & 52,95 & 41,88 & 46,85 \\
\hline Espessura da Folha $(\mu \mathrm{m})$ & 43,61 & 45,31 & 43,39 & 27,59 & 34,11 & 55,20 & 36,97 & 31,64 \\
\hline
\end{tabular}

IBI: Ibitinga. JUP: Jupiá. TRI: Três Irmãos. NAV: Nova Avanhandava. PRO: Promissão. SAG: Salto Grande.

$\underline{1}$ acesso $1 . \quad \underline{2}$ acesso 2 .

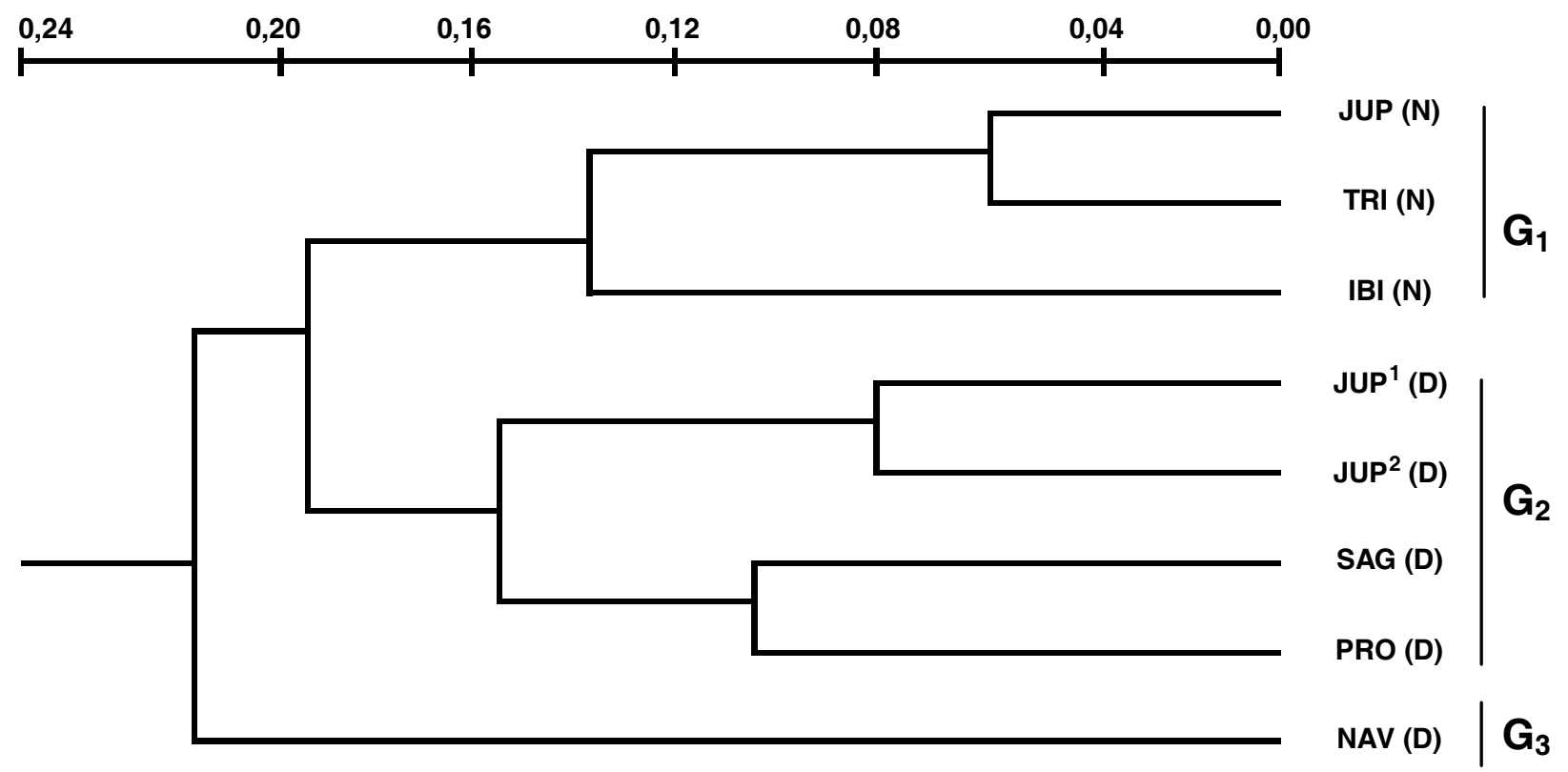

Figura 3 - Dendrograma resultante da Análise de Agrupamento dos sete caracteres anatômicos quantitativos do limbo foliar, utilizandose a Distância Euclidiana Média entre os oito acessos de Egeria. JUP: Jupiá, TRI: Três Irmãos, IBI: Ibitinga, SAG: Salto Grande, PRO: Promissão e NAV: Nova Avanhandava. (N): Egeria najas e (D): Egeria densa. 
No grupo 1 (Figura 3), os acessos de E. najas coletados em Jupiá e Três Irmãos apresentaram maior similaridade, provavelmente pela proximidade de localização entre esses reservatórios no rio Tietê, situados na região extremo oeste do Estado de São Paulo, próximo à bacia do rio Paraná (Figura 1). Por sua vez, o acesso coletado em Ibitinga uniu-se aos outros dois acessos desse grupo em um nível de similaridade menor, possivelmente devido à maior distância geográfica (Figura 1). Contudo, os acessos desses três reservatórios formaram um grupo representado apenas por E. najas, apesar de estarem localizados em regiões com latitudes e longitudes diferentes (Tabela 1).

De acordo com Mori et al. (1999), a variabilidade genética observada nas populações de E. najas coletadas no reservatório de Jupiá e rios afluentes pode ser originária de propagação sexuada entre indivíduos, devido à alta densidade populacional, que favorece a polinização cruzada, e/ou oriunda de material vegetativo variável, de diferentes origens dos afluentes da bacia do rio Paraná. Esses mesmos autores constataram diferenças genéticas entre os acessos coletados no reservatório de Jupiá, no rio Tietê, no canal de Pereira Barreto e no rio São José dos Dourados.

No grupo 2 (Figura 3), os acessos de E. densa coletados em Jupiál e Jupiá ${ }^{2}$ apresentaram alta similaridade, constituindo um subgrupo, devido à sua localização no mesmo reservatório. Os acessos coletados em Salto Grande e Promissão também mostraram similaridade e formaram outro subgrupo, apesar de o reservatório de Salto Grande estar localizado fora da bacia do rio Tietê e, portanto, mais distante geograficamente do reservatório de Promissão (Figura 1), porém localizado em região com longitude próxima à do outro reservatório (Tabela 1). Esses dois subgrupos formados uniram-se em um nível de similaridade menor, possivelmente devido à maior distância geográfica, uma vez que se localizam em latitudes e longitudes diferentes. Conseqüentemente, esses reservatórios podem apresentar microambientes distintos, estando submetidos à influência antrópica variada, o que pode modificar as características anatômicas foliares dos acessos, acarretando formação de subgrupos, além da possibilidade de ocorrência de reprodução sexuada. Entretanto, os acessos desses quatro reservatórios formaram um grupo representado apenas por E. densa, apesar de estarem localizados em regiões diferentes (Tabela 1 e Figura 1).

O grupo 3 foi formado apenas pelo acesso de E. densa coletado em Nova Avanhandava (Figura 3), provavelmente pela localização intermediária desse reservatório em relação aos demais (Figura 1), os quais constituíram os grupos de E. najas (grupo 1) e E. densa (grupo 2).

Martins et al. (2003) constataram que a porcentagem de locos polimórficos observada para a população de E. najas no reservatório de Jupiá foi menor do que a observada em $E$. densa, evidenciando que E. najas foi menos variável geneticamente que $E$. densa.

$\mathrm{O}$ aumento da variabilidade genética dentro da população de E. densa e E. najas, possivelmente, deve-se ao fato de estas espécies serem nativas da bacia do rio Paraná e, ocasionalmente, apresentarem reprodução por sementes (Cook \& Urmi-König, 1984; Martins et al., 2003).

Os reservatórios de Promissão (grupo 2) e de Nova Avanhandava (grupo 3) apresentam proximidade geográfica no rio Tietê (Figura 1); entretanto, os acessos de E. densa coletados nesses dois reservatórios mostraram alta dissimilaridade, constituindo dois grupos distintos (Figura 3), corroborando os resultados obtidos por Martins et al. (2003), referentes à caracterização genética de acessos de $E$. densa e E. najas coletados em reservatórios hidrelétricos do Estado de São Paulo, utilizando a técnica de RAPD.

Weiss et al. (1997), também utilizando RAPD, concluíram que E. densa é diferente de Elodea canadensis, constatando também a ocorrência de pequenas diferenças genéticas entre os acessos de E. densa coletados na Califórnia. Kadono et al. (1997), estudando a variabilidade genética pelo método isoenzimático em acessos de E. densa e Elodea nuttalli coletados em vários locais do Japão, não encontraram diferenças genéticas, sugerindo que os acessos de egéria eram provenientes de um mesmo clone introduzido no Japão.

Na Tabela 3 são apresentados os coeficientes de correlação entre os sete caracteres 
anatômicos quantitativos do limbo foliar e os componentes principais $\left(\mathrm{Y}_{1}\right.$ e $\left.\mathrm{Y}_{2}\right)$. No conjunto, esses componentes foram responsáveis por 83,94\% da informação (variância) acumulada pelos caracteres avaliados, constituindo-se em indicadores eficientes de dissimilaridade, comportando-se, portanto, como caracteres diferenciadores entre os acessos estudados. A intensidade da contribuição desses caracteres para a discriminação dos acessos está relacionada à ocorrência de maiores valores absolutos em $\mathrm{Y}_{1}$ e $\mathrm{Y}_{2}$.

Verifica-se na Tabela 3 que, para o primeiro componente principal $\left(\mathrm{Y}_{1}\right)$, a porcentagem de feixe vascular da nervura central foi o caráter anatômico quantitativo que mais contribuiu para a discriminação dos oito acessos de Egeria, enquanto as porcentagens de epiderme da face abaxial da nervura central e de epiderme das faces adaxial e abaxial da região situada entre a nervura e o bordo foliar apresentaram menor contribuição. Quanto ao segundo componente principal $\left(\mathrm{Y}_{2}\right)$, apenas a espessura da folha mostrou poder de discriminação satisfatório.

A dispersão gráfica (Figura 4), resultante da Análise de Componentes Principais, também mostrou a formação de três grupos, com relação aos oito acessos das duas espécies de Egeria estudadas, revelando haver boa concordância entre esses resultados e aqueles obtidos da Análise de Agrupamento (Figura 3).

Observando o componente principal $\mathrm{Y}_{1}$ (Tabela 3) e a dispersão gráfica (Figura 4), verificou-se que os acessos de E. najas coletados nos reservatórios do grupo 1 (Três Irmãos, Jupiá e Ibitinga) apresentaram menores valores de porcentagem de feixe vascular da nervura central que os demais acessos de E. densa formadores dos grupos 2 e 3, excetuando-se o acesso do reservatório Jupiá ${ }^{1}$ do grupo 2, provavelmente por estar localizado na mesma região daquele do grupo 1 (Tabela 2). Quanto à porcentagem de epiderme da região situada entre a nervura e o bordo foliar, os acessos de E. najas do grupo 1 apresentaram maiores valores para a face adaxial e menores para a face abaxial (Tabela 2). A porcentagem de epiderme da face abaxial da nervura central dos acessos de E. najas do grupol revelou maiores valores que os demais acessos de E. densa constituintes dos grupos
2 e 3, excetuando-se o acesso do reservatório Salto Grande do grupo 2, que apresentou maior valor que o acesso do reservatório Jupiá do grupo 1 (Tabela 2), possivelmente por estar localizado em região com latitude e longitude diferentes (Figura 1).

Observando o componente principal $\mathrm{Y}_{2}$ (Tabela 3) e a dispersão gráfica (Figura 4), constatou-se que os acessos de E. densa coletados nos reservatórios do grupo 2 (Jupiá ${ }^{1}$, Jupiá $^{2}$, Salto Grande e Promissão) apresentaram menores valores da espessura da folha que os acessos de E. najas do grupo 1 e também de $E$. densa do grupo 3 (Tabela 2).

Tabela 3 - Coeficientes de correlação entre os caracteres anatômicos quantitativos do limbo foliar de oito acessos de Egeria densa e E. najas e os dois primeiros componentes principais $\left(\mathrm{Y}_{1}\right.$ e $\left.\mathrm{Y}_{2}\right)$

\begin{tabular}{|c|c|c|}
\hline Caráter & $\mathrm{Y}_{1}$ & $\mathrm{Y}_{2}$ \\
\hline$\%$ Epiderme Face Adaxial (NC) & $-0,2181$ & $-0,7592$ \\
\hline$\%$ Epiderme Face Abaxial (NC) & $-0,6939$ & 0,3343 \\
\hline \% Parênquima (NC) & 0,5663 & 0,7342 \\
\hline$\%$ Feixe Vascular (NC) & 0,9703 & $-0,2123$ \\
\hline \% Epiderme Face Adaxial (RNB) & $-0,6737$ & $-0,2130$ \\
\hline$\%$ Epiderme Face Abaxial (RNB) & 0,6728 & 0,2115 \\
\hline Espessura da Folha (RNB) & $-0,0680$ & 0,9728 \\
\hline \% Informação Retida & 48,32 & 35,62 \\
\hline \% Informação Acumulada & 48,32 & 83,94 \\
\hline
\end{tabular}

NC: nervura central e RNB: região entre a nervura central e o bordo do limbo foliar.

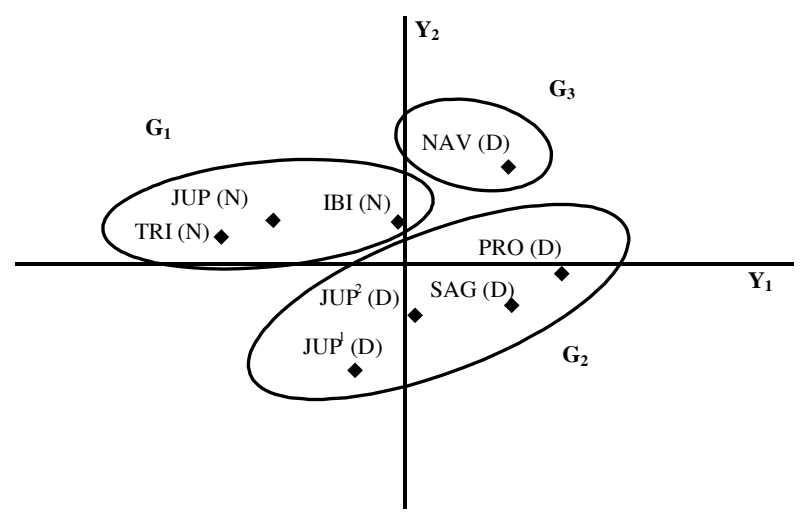

Figura 4 - Dispersão gráfica dos oito acessos de Egeria, utilizando-se os dois primeiros componentes principais $\left(\mathrm{Y}_{1}\right.$ e $\mathrm{Y}_{2}$ ), para o conjunto dos sete caracteres anatômicos quantitativos do limbo foliar. JUP: Jupiá, TRI: Três Irmãos, IBI: Ibitinga, SAG: Salto Grande, PRO: Promissão e NAV: Nova Avanhandava. (N): Egeria najas e(D): Egeria densa. 
As diferenças anatômicas quantitativas encontradas entre os acessos permitiram diferenciar E. densa de E. najas, constituindo grupos distintos. Contudo, ainda não é bem conhecida a influência dos caracteres anatômicos do limbo foliar sobre a eficácia e seletividade de herbicidas no controle destas espécies, havendo a necessidade de realizar mais estudo sobre esse assunto.

Tanaka et al. (2002), avaliando os efeitos do herbicida fluridone (1-methyl-3-phenyl-5[3-(trifluoromethyl)phenyl]-4(H)-pyridinone) no controle de E. densa e E. najas, observaram que E. najas mostrou-se mais sensivel que E. densa; entretanto, ao final do experimento os niveis de controle das duas espécies foram semelhantes. Netherland et al. (2000) verificaram a tolerância ao herbicida fluridone numa população de plantas de Hydrilla verticillata coletadas em diversos lagos da região central da Flórida, exigindo-se doses mais elevadas para a maior eficiência do herbicida.

De acordo com Marcondes et al. (2003b), a exposição contínua ao herbicida fluridone, por um período de 130 dias, reduziu significativamente a biomassa de E. densa e E. najas, atingindo niveis de controle superiores a 98,3\%. No entanto, quando cessou o efeito do fluridone, ocorreu a recolonização do ambiente pelas espécies estudadas.

Com base nos resultados obtidos, verificou-se que os caracteres anatômicos quantitativos do limbo foliar são discriminatórios e permitiram estabelecer diferenças entre os acessos de E. najas (grupo 1) e os de E. densa (grupos 2 e 3). As diferenças anatômicas encontradas, principalmente, quanto à \% feixe vascular e \% epiderme da face abaxial da nervura central, além da \% epiderme de ambas as faces da região situada entre a nervura e o bordo do limbo foliar, podem auxiliar na correta identificação dessas espécies da flora daninha em levantamentos fitossociológicos realizados em reservatórios hidrelétricos.

Os caracteres anatômicos avaliados não apresentaram relação com a suscetibilidade e resistência dos acessos a herbicidas. Portanto, os estudos referentes à determinação de acessos suscetiveis, tolerantes ou resistentes a herbicidas, e a eventual relação da anatomia nesse comportamento devem ser incrementados, visando o conhecimento da seletividade para essas espécies.

\section{LITERATURA CITADA}

BOWMER, K. H.; JACOBS, S. W. L.; SAINTY, G. R. Identification, biology and management of Elodea canadensis, Hidrocharitaceae. J. Aquatic Plant Manag., v. 33, p. 13-19, 1995.

CARDOSO, L. R. et al. Variabilidade genética de acessos de aguapé coletados no Estado de São Paulo. Planta Daninha, v. 20, p. 1-5, 2002. Edição especial

COOK, C. D. K.; URMI-KÖNIG, K. A revision of the genus Egeria (Hydrocharitaceae). Aquatic Bot., v. 19, p. 73-96, 1984.

FERREIRA, E. A. et al. Estudos anatômicos de folhas de plantas daninhas. II. Bidens pilosa, Emilia sonchifolia, Agerantum conyzoides e Sonchus asper. Planta Daninha, v. 20, n. 3, p. 327-335, 2002.

GERRITS, P. O. The application of glycol metacrylate in histotechnology: same fundamental principles. Germany: Leica Gmgh, 1991. 80 p.

HOYER, M. V. et al. Florida freshwater plants: a handbook of common aquatic plants in Florida lakes. Gainesville: University of Florida, 1996. 264 p.

JOHANSEN, D. A. Plant microtechnique. New York: McGraw-Hill Book, 1940. 523 p.

KADONO, Y.; NAKAMURA, T.; SUZUKI, T. Genetic uniformity of two aquatic plant, Egeria densa Planch. and Elodea nuttallii (Planch.) St. John, introduced in Japan.

Japan J. Limnol., v. 58, p. 197-203, 1997.

KISSMANN, K. G.; GROTH, D. Plantas infestantes e nocivas. 2.ed. São Paulo: BASF, 1997. p. 290-293.

MARCONDES, D. A. S.; MUSTAFA, A. L.; TANAKA, R. H. Estudos para manejo integrado de plantas aquáticas no reservatório de Jupiá. In: THOMAS, S. M.; BINI, L. M. (Eds.). Ecologia e manejo de macrófitas aquáticas. Maringá: EDUEM, 2003a. p. 299-317.

MARCONDES, D. A. S. et al. Eficiência de fluridone no controle de plantas aquáticas submersas no reservatório de Jupiá. Planta Daninha, v. 21, p. 69-77, 2003b. Edição especial

MARTINS, D. et al. Caracterização genética de acessos de egéria (Egeria spp.) coletados no Estado de São Paulo utilizando RAPD. Planta Daninha, v. 21, p. 1-6, 2003. Edição especial 
MORI, E. S. et al. Caracterização genética de populações de Egeria najas presentes no reservatório de Jupiá e rios afluentes. Planta Daninha, v. 17, n. 2, p. 217-225, 1999.

NETHERLAND, M. et al. Differential response of hydrilla to fluridone. In: AQUATIC PLANT MANAGEMENT SOCIETY, FORTIETH ANNUAL MEETING, 24., 2000, San Diego. Abstracts... San Diego: Aquatic Plant Management Society. 2000. p. 49.

O`BRIEN, T. P.; FEDER, N.; McCULLY, M. E. Polychromatic staining of plant cellular wall by toluidine blue. Protoplasma, v. 59. p. 368-373, 1964.

PROCÓPIO, S. O. et al. Estudos anatômicos de folhas de plantas daninhas de grande ocorrência no Brasil. III. Galinsoga parviflora, Crotalaria incana, Conyza bonariensis e Ipomoea cairica. Planta Daninha, v. 21, n. 1, p. 1-9, 2003a.

PROCÓPIO, S. O. et al. Anatomia foliar de plantas daninhas do Brasil. Viçosa, MG: Universidade Federal de Viçosa, 2003b. v. 1. 118 p.

ROBERTS, D. E.; CHURCH, A. G.; CUMMINS, S. P. Invasion of Egeria into the Hawkesbury-Nepean River, Australia. J. Aquatic Plant Manag., v. 37, p. 31-34, 1999.

ROHLF, F. J. Adaptative hierarchical clustering schemes. Syst. Zool., v. 19, p. 58-82, 1970.

SCULTHORPE, C. D. The biology of aquatic plants. London: E. Arnold, 1967. 610 p.

SNEATH, P. H. A.; SOKAL, R. R. Numerical taxonomy. San Francisco: W. H. Freemam, 1973. 573 p.
SOLYMOSY, S. L.; GANGSTAD, E. O. Nomenclature, taxonomy and distribution of Egeria and Elodea. Hyacinth Control J., v. 12, p. 3-5, 1974.

SOUZA, L. A. et al. Morfologia e anatomia vegetal: técnicas e práticas. Ponta Grossa: Universidade Estadual de Ponta Grossa, 2005. 194 p.

TANAKA, R. H. Prejuízos provocados pelas plantas aquáticas. In: WORKSHOP DE CONTROLE DE PLANTAS AQUÁTICAS, 1998, Brasília-DF. Resumos... Brasília: IBAMA, 1998. p. 36-38.

TANAKA, R. H. Eficácia de fluridone para o controle de Egeria ssp. em caixas d'água e em represa de pequeno porte sem fluxo de água. 2000, 157 f. Dissertação (Mestrado em Agricultura) - Universidade Estadual Paulista, Botucatu, 2000.

TANAKA, R. H. et al. Avaliação de herbicidas para o controle de egéria em laboratório, caixa d'água e represa sem fluxo de água. Planta Daninha, v. 20, p. 73-81, 2002. Edição especial.

THOMAZ, S. M. Fatores ecológicos associados à colonização e ao desenvolvimento de macrófitas aquáticas e desafios de manejo. Planta Daninha, v. 20, p. 21-33, 2002. Edição especial

WEISS, A. et al. Survey of Egeria densa accessions for genetic similarity by Random Amplified Polymorphic DNA Analysis (RAPDs). Aquatic Weed Control Invest., p. 69-73, 1997.

YEO, R. R.; FALK, R. H.; THURSTON, J. R. The morphology of Hydrilla (Hydrilla verticillata (L. f.) Royle). J. Aquatic Plant Manag., v. 22, p. 1-17, 1984. 